\title{
Commentary
}

\section{Adding Pieces to the Puzzle: A Commentary}

\author{
Beverly D. Ulrich and Masayoshi Kubo \\ Division of Kinesiology, University of Michigan, 401 Washtenaw Ave, Ann Arbor, \\ MI 48109-2214, USA
}

In Jensen's theme paper she did an excellent job of outlining and explaining ways in which motor developmentalists can use the tools of biomechanics and engineering to address research questions. We extend her thorough efforts in two ways. First, we highlight the communication value provided by the precision of biomechanics terminology, and second, we reinforce the need to embed motion (the kinematics and kinetics) in context and function in order to understand the complexity of behaviour. We illustrate how one biomechanical tool, mechanical models, can facilitate this and provide insight to the development of control. Copyright (C) 2005 John Wiley \& Sons, Ltd.

Key words: dynamic solutions; complex systems; funtional behaviour

One of the most challenging aspects of developmental science for those of us studying the motor behaviour of our participants is to collect accurate, valid, and precise data that reflects their activity. In the early part of the 20th century clever people like Shirley (1931) oiled the bottoms of toddlers' feet and had them walk over paper to track their gait patterns. In the 1960s and 1970s Roberton and colleagues (Roberton, 1978; Roberton et al., 1979) used $16 \mathrm{~mm}$ film to record children performing an overarm throw, then they laboriously digitized, frame by frame, the qualitative changes in patterns of limb movement over developmental time. These scientists asked important developmental questions, found appropriate ways to answer them, and helped us all learn about the emergence of skills. But, their efforts were also labour intensive and fairly daunting for all but the most patient and persistent scholars.

Fortunately, our colleagues in biomechanics and engineering have devised many new and much more sophisticated ways to collect a variety of types of data, both in the laboratory and in field settings, that have made the goal of detailed motion description more accessible. Today we can choose, for example, from many motion analysis systems to collect joint position data at high frame rates in real time and in three dimensions. This is not to suggest it is easy-it is not. Jensen shielded us from the details of the training required to use the principles, techniques and equipment she described as she so elegantly outlined ways in which biomechanics can contribute to addressing the puzzles of motor development. We fully concur that this area has much to offer the motor 
developmentalist. It is a wise investment of time to learn how to use these tools and to understand the biomechanical principles that underlie their use as well as their limitations. Collaborations with experts in this area can also facilitate the process.

Jensen did an excellent job of organizing a set of questions for motor developmentalists that we believe should ring true to developmental scientists, whether they focus on movement, as she did, or not. One could substitute cognitive or social for motor and be comfortable with this set of issues from which to approach understanding the performance repertoire. But, as she aptly pointed out, in the motor realm researchers have a more accessible window on performance than most. We can access the kinematics and kinetics of actions as they unfold from the earliest activation of muscles, to the trajectory through which the body segments move, to the endpoint success or lack thereof.

We would add to Dr Jensen's list of ways in which biomechanical tools are helpful by noting that the terminology used provides a common language that can aid communication. Dialogue among scientists from varied disciplines, as well as physicians, therapists, and educators can flow more smoothly and seamlessly if we use terms with common and well-defined meanings. Within the area of motor behaviour science we too often become lax about terms we use, sometimes using coordination and control, for example, as if they described a single characteristic or, as Jensen acknowledged, we fail to agree on the definition of a term used as commonly as 'skill'.

The language of biomechanics, per se, is functional and refreshingly precise. It seems reasonable also to reinforce that kinematics describe the motion of limbs, centres of mass, etc., and kinetics reflect the forces or torques that underlie the trajectories observed. Description itself does not provide explanation and motion is not synonymous with behaviour. Behaviour is complex, it occurs in context, and is impacted by multiple subsystems in addition to the biology and mechanics of our systems. That does not negate the enormous contribution biomechanics tools can make, but it reminds us that at some point motion must be contextualized to link understanding to function. A recent study we conducted illustrates this point. We used the tools of biomechanics to examine the gait patterns of preadolescent children with Down's syndrome (DS) and to compare their behaviour to that of children with typical development (TD) as they walked on a treadmill and over ground (Ulrich et al., 2004). Specifically, we used a mechanical model developed by Holt and colleagues that depicts the motion of the centre of mass during the stance phase of walking as an inverted pendulum, rotating over the ankle as the body moves forward (see Figure 1). The net motion of the centre of mass is a function of the torques due to gravity, angular impulse due to active muscle contraction, global stiffness and damping. The model takes into account body mass and segment lengths, among other parameters, and can be used to estimate the magnitude of dynamic resources used. Our goal was to understand how systems with different properties (i.e. children with DS and TD) use their resources, in particular stiffness and impulse, to produce the same behaviour-walking.

Results showed significant differences between the groups; for example, children with DS generated significantly higher levels of stiffness than their peers with TD, but only when walking on a treadmill, not when walking over ground. The model estimated stiffness as a function of higher step frequency. Yet this model did not explain why their step frequency was higher than their body size and physics principals would have predicted, only on the treadmill. We argued that their behaviour emerged as a strategy children with DS discovered in order 


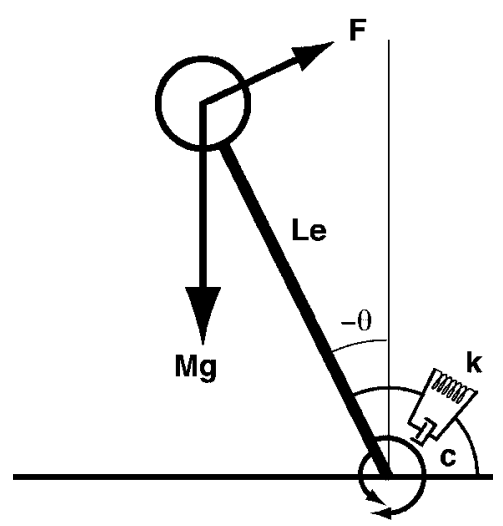

Figure 1. The inverted pendulum model of walking, where $M$ is the mass, $g$ is the gravitational constant, $F$ is the driving force generated by active muscle contraction. $k$ is the coefficient of global stiffness and $c$ is the damping coefficient. Le is the simple pendulum equivalent length calculated based on Obusek et al. (1995).

to stabilize their limbs, particularly when they perceived their stability to be challenged. This occurred when they experienced a novel context, the treadmill. The ligaments of children with DS are inherently lax, making their joints less stable and more difficult to control than the tighter joints of their peers with TD. By increasing the tension in the muscles surrounding their joints when they perceived their control to be threatened they selected an optimal solution, given the task, the context, and their own complex systems. Our conclusions remain hypothetical and require experiments, currently underway, to validate. We also believe the mechanics mattered; behaviour was explained, to a large degree, by the mechanical model and the use of this model in varied contexts provided us with insight that a more reductionist approach could not.

Mechanical models provide a valuable tool because they compress high dimensionality and reflect the essence of the system's behaviour. Functional behaviour involves the interactive convergence of many intrinsic and extrinsic subsystems and results in shifting patterns of coordination due to mechanics, experience, task demands, social constraints, and so on. A heuristic often used to depict the shifts in behaviour over time is the epigenetic landscape of hills, valleys, and bifurcations (Muchinsky et al., 1996). Models can reflect the essence of behaviour at some point or area within that landscape, but when parameters change sufficiently the behaviour may shift into a new dynamic solution, thus requiring the model to be adapted as well. Our attempts to apply the inverted pendulum model to the earliest walking patterns of infants with TD provided insight that the simple efficient planer motion of practiced walkers was not the solution to which new walkers were attracted. When we studied toddlers able to walk only six to eight steps independently our model solution suggested that toddlers produced a net impulse at the onset of swing in the direction opposite to forward progression (Holt et al., submitted). These values emerged because the model is based on an assumption that the centre of mass motion occurs principally in the sagittal plane and that the impulse for forward progression occurs within a particular window of the stride cycle. In reality, toddlers show a vast distribution of impulse timing, and motion in other planes was observed as well, particularly in the medio-lateral direction. While this resolved quickly, 
within a month of walking experience, it illustrates the process by which behaviour is transformed into efficient motion, that is, by exploring a host of options that are within the functional, if not optimal, workspace.

In summary, we agree wholeheartedly with Jensen's assessment that biomechanics and engineering tools and principals have much to offer the motor developmentalist as well as others for whom motion provides a window to the processes that underlie behaviour. The trick is to refrain from assuming that description is explanation. If explanation is the goal then we must look further, for the insight, as Jensen suggested, that these detailed and carefully measured dependent variables provide in our efforts to understand the processes that underlie the emergence of and change in patterns of behaviour.

\section{ACKNOWLEDGEMENTS}

This work was supported in part by National Institutes of Health Grant R01 HD 42728 awarded to the first author.

\section{REFERENCES}

Holt KG, Saltzman E, Ho Chia-Ling, Kubo M, Ulrich BD. Discovery of the pendulum and spring dynamics in the early stages of walking, submitted for publication.

Jensen JL. 2005. The puzzles of motor development: how the study of developmental biomechanics contributes to the puzzle solutions. Infant and Child Development 14: 501-511.

Muchinsky M, Gershkoff-Stowe L, Cole E, Thelen E. 1996. The epigenetic landscape revisited: a dynamic interpretation. In Advances in Infancy Research, vol. 10, Rovee-Collier C, Lipsitt LP (eds). Ablex: Norwood, NJ; 121-159.

Obusek JP, Holt KG, Rosenstein RM. 1995. The hybrid mass-spring pendulum model of human leg swinging: stiffness in the control of cycle period. Biological Cybernetics 73: 139-147.

Roberton MA. 1978. Longitudinal evidence for developmental stages in the forceful overarm throw (Mise en evidence par etude longitudinale des stades de developpementmoteur dans le lancer a bras casse a pleine force). Journal of Human Movement Studies 4(3): 167-175.

Roberton MA, Halverson LE, Langendorfer S, Williams K. 1979. Longitudinal changes in children's overarm throw ball velocities. Research Quarterly 50(2): 256-264.

Shirley MM. 1931. The First Two Years: A Study of 25 Babies, Volume 1: Postural and Locomotor Development. University of Minnesota Press: Minneapolis, MN.

Ulrich BD, Haehl V, Buzzi UH, Kubo M, Holt KG. 2004. Modeling dynamic resource utilization in populations with unique constraints: preadolescents with and without Down syndrome. Human Movement Science 23: 133-156. 